\title{
O programa de monitoramento da biodiversidade e do uso sustentável de recursos naturais -ProBUC - como alternativa de monitoramento comunitário amazônico.
}

\author{
Daniel Carneiro Costa', Guillaume Antoine Marchand ${ }^{2}$ \\ ' Mestrando do Programa de Pós-graduação em Ciências Ambientais e Sustentabilidade na Amazônia, Universidade Federal do Amazonas, \\ Manaus - AM - Brasil \\ ${ }^{2}$ Doutor em Geografia pela Universidade de Paris III Sorbonne-Nouvelle, Professor visitante do Centro de Ciências do Ambiente, Univer- \\ sidade Federal do Amazonas, Manaus - AM - Brasil
}

\begin{abstract}
Resumo
O ProBUC - programa de monitoramento da biodiversidade e do uso sustentável de recursos naturais - é apresentado neste trabalho como uma inovação no processo metodológico de monitoramento comunitário no estado do Amazonas. Seu surgimento visa subsidiar a gestão de unidades de conservação estaduais amazonenses. Esta necessidade resulta de um processo maior de aperfeiçoamento da gestão das áreas protegidas em todo o globo, a partir de uma avaliação que considere a realidade de cada bioma e a valorização de suas especificidades. Neste contexto, cresceram, nos últimos anos, as experiências de monitoramento comunitário, especialmente nos países tropicais, onde o monitoramento convencional normalmente é mais caro e não conta com adequado suporte orçamentário e técnico dos governos nacionais. Assim, esta modalidade alternativa de monitoramento comunitário visa incorpora a participação das comunidades locais em todas as suas etapas de implantação, ou seja, da definição de prioridades de espécies e recursos a serem monitorados à interpretação dos resultados com o fito de promover um diagnóstico que possa contribuir com a gestão da área protegida. Pretende-se conhecer a abrangência e metodologia do ProBUC para saber se realmente corresponde a um programa de monitoramento comunitário. Utiliza-se da pesquisa teórica e observações diretas neste artigo. As controvérsias e aspectos complementares entre as modalidades de monitoramento convencional e comunitário são expostas por autores internacionais até se chegar à exposição da proposta do programa estadual amazonense. Outrossim, as principais conclusões de 2 eventos avaliativos sobre este programa são apresentadas, as quais mostram que ainda são necessários diversos ajustes para o funcionamento adequado do programa. Por fim, constata-se que sua implantação nas 3 unidades de conservação precisa de uma avaliação para conhecer sua viabilidade e capacidade de envolvimento comunitário local.
\end{abstract}

Palavras-chaves:

\begin{abstract}
The ProBUC - monitoring program of biodiversity and the sustainable use of natural resources - is presented in this paper as a methodological innovation in the process of community monitoring in the state of Amazonas. Its appearance is intended to subsidize the management of Amazonian state conservation units. This requirement results in a larger process of improving the management of protected across the globe, from a review to consider the reality of each biome and appreciation of their specific areas. In this context, increased in recent years, the experiences of community monitoring, especially in tropical countries, where conventional monitoring is typically more expensive and does not have adequate budgetary and technical support from national governments. Thus, this alternative form of community monitoring aims incorporates the participation of local communities in all stages of implementation, the prioritization of species and resources to monitor the interpretation of the results with the aim of promoting a diagnosis that can contribute to the management of the protected area. It is intended to meet the scope and methodology of ProBUC to know if it really corresponds to a community monitoring program. It is used in theoretical research and direct observations in this article. Controversies and complementary aspects between conventional methods of monitoring and community are exposed by international authors to get exposure to the proposal of Amazonas state program. Furthermore, the main findings of two evaluation events on this program are presented, which show that many adjustments for proper functioning of the program are still required. Finally, it appears that their deployment in 3 conservation units need a review to know its sustainability and local community involvement.
\end{abstract}

Keywords: 


\section{INTRODUÇÃO}

A adoção de métodos de gestão e avaliação das unidades de conservação (UCs) alheios às especificidades das realidades amazônicas resultou em certa ineficiência de sua aplicação e na importância de se desenvolverem métodos alternativos. Por mais bem elaborados que tenham sido e com alcance internacional, os sistemas criados para acompanhar e monitorar a gestão das UCs não incorporaram aspectos fundamentais do complexo contexto amazônico. A diversidade de ecossistemas relacionados requer o conhecimento de particularidades essenciais para seu funcionamento.

Outra crítica aos atuais padrões de avaliação de gestão das UCs, especialmente na Amazônia, refere-se à sua análise sobre a dimensão da apropriação e do uso sustentável dos recursos naturais e da biodiversidade pelas populações locais. Sobretudo, "[...] como parte dos processos das dinâmicas que regem as UCs, ou seja, nas equações socioambientais de sustentabilidade desses sistemas". (MARINELLI et al, 2011, p. 6). Em geral, os mecanismos previstos na legislação e nos métodos tradicionais não foram suficientes para estimular e detalhar o nível desta gestão compartilhada entre os atores sociais afins. Este processo "[...] impede que os resultados obtidos com essas metodologias se desdobrem em ações de manejo mais estratégicas e que atendam necessidades específicas do contexto de cada UC". (idem, p. 10). Neste contexto, o monitoramento desempenha uma tarefa estratégica para a obtenção de dados que serão fundamentais para a formulação de políticas e diretrizes para o interior das UCs. Especialmente nos países tropicais, que apresentam a maior parte de biomas preservados em escala global, a participação comunitária tornou-se uma necessidade não apenas para a gestão como para a própria atividade do monitoramento, uma vez que sua presença no interior destas áreas protegidas reflete-se num conhecimento significativo sobre a biodiversidade ameaça.

\section{METODOLOGIA}

Esta pesquisa foi realizada por meio de referencial teórico referente a autores que debatem as diferenças entre monitoramento convencional e comunitário, observando a concretização desta segunda modalidade à luz de experiências internacionais. Num segundo momento, o programa amazonense ProBUC é apresentado dentro da metodologia participativa proposta. As observações diretas das duas reuniões de avaliação do ProBUC, realizadas em 2010 e 2013, complementam o percurso metodológico deste artigo. Elas resultaram numa socialização de análises sobre o estágio do programa conforme a ótica de todos os envolvidos (caso do primeiro workshop) ou serviram para aprofundar aspectos tomados como mais técnicos, restringindo o público participante (caso do segundo workshop).

\section{RESULTADOS E DISCUSSÕES}

\subsection{Do monitoramento convencional ao monitoramento comunitário na perspectiva da gestão de áreas protegidas.}

No contexto da gestão de áreas protegidas, o monitoramento constitui ferramenta importante, responsável pela identificação e diagnóstico de algo ao longo de um tempo e espaço determinados. Segundo Junior et al (2011, p.11) monitorar é "acompanhar, diagnosticar e identificar tendências de algo ao longo do tempo [...]", permitindo "[...] compreender o estado da biodiversidade, a forma com esta está sendo usada, seus níveis de ameaça e potencialidades [...]"(idem p.11). Em outros termos, consiste na "[....] observação repetida para fins específicos, de um ou mais elementos do ambiente, de acordo com horários pré estabelecidos, no espaço e no tempo, utilizando métodos de coleta de dados comparáveis [...]" (HOCKINGS, 2000, p.3). Desta maneira, o monitoramento da biodiversidade torna-se um aliado da gestão de áreas protegidas, a exemplo das unidades de conservação brasileiras, proporcionando informações para o planejamento da gestão e para auxiliar as pesquisas científicas. Esta atividade há aproximadamente 40 anos vem sendo realizada com mais freqüência (GLASSON, THERIVEL e CHADWICK 1994) em decorrência da constatação dos impactos crescentes da ação antrópica sobre os recursos naturais e a biodiversidade. Construiu-se, paulatinamente, o consenso acerca da necessidade de se compreender ao máximo as dinâmicas dos sistemas complexos que organizam os ecossistemas. Por meio do monitoramento, possibilita-se avaliar o estado de um recurso ou sistema, 
municiando a gestão da área protegida ou especialmente da UC de prováveis eventos (CUNDILL e FABRICIUS, 2009). Em meados dos anos de 1990, a gestão dos projetos ambientais passou a incorporar o monitoramento como essencial (LAMMERTS van BUEREN e BLOM, 1997). Juntamente com o processo de avaliação da gestão das áreas protegidas, as bases conceituais do monitoramento se expandiram expressivamente nos últimos 10 anos (CUNDILL e FABRICIUS, 2009).

Como pontuam Garcia e Lescuyer (2008), os sistemas de monitoramento funcionam por meio de aparelhos que incorporam inovações tecnológicas, as quais permitem a observação das mudanças tanto físicas quanto sociais de um ambiente considerado. São usados para armazenar e ordenar informações estratégicas que dizem respeito às consequências negativas e positivas da implementação de projetos. Por esta razão, muito além de se constituírem como fonte de conhecimento da diversidade biológica local para auxiliar a gestão de recursos naturais, os sistemas de monitoramento podem sinalizar para a gestão mudar os objetivos e rumos pré-estabelecidos, influindo diretamente na tomada de decisões. $\mathrm{O}$ monitoramento, segundo os autores supracitados, permite a criação de indicadores, os quais facilitam a percepção ambiental e criação de expectativas em relação ao ambiente natural e às dinâmicas das populações locais e demais atores sociais envolvidos no contexto. No entanto, diversas dificuldades foram detectadas ao se inserir as atividades de monitoramento em escalas distintas daquelas para as quais foram inicialmente programadas. Questionamentos sobre a consistência científica foram realizados em relação ao seu uso diário por projetos de gestão de recursos naturais. Há vasta literatura que trata da introdução, funcionamento e avaliação de sistemas de monitoramento. Percebeu-se, então, que a incorporação das populações locais nos sistemas de monitoramento poderia proporcionar ganhos às suas finalidades por causa do conhecimento local que possuem. Uma importante constatação para esta mudança de posição científica só ocorreu porque

[...] Em alguns casos, as percepções sobre a situação dos recursos naturais são maiores entre usuários do que entre os cientistas. Os produtores percebem com antecedência, pelo contato próximo, os sinais da superexploração, da distribuição restrita e quando o acesso aos recursos é limitado [...] (REBÊLO e PEZZUTI, 2000).

Segundo Sheil e Lawrence (2004) outro fator relevante para a mudança de paradigma da atividade de monitoramento foi a constatação da aceleração da extinção de espécies biológicas pouco investigadas, em diversas partes do mundo, particularmente nas regiões tropicais. Muitos cientistas locais propuseram, desde então, a incorporação das populações locais ao trabalho de cogestão de áreas protegidas. As populações locais tornaram-se parte do processo de coleta taxonômica, estimativa de espécies biológicas, ampliando a sensibilização social e comunitária para a conservação dos recursos naturais e da biodiversidade. Esta nova metodologia viabilizou políticas locais e nacionais que buscam a redução dos impactos ambientais sobre as áreas protegidas. Neste cenário, o aproveitamento das experiências comunitárias para realização das pesquisas biológicas ocorridas nos países tropicais é uma realidade crescente há pouco tempo. Os resultados destas abordagens são impressionantes. Estas comunidades, inicialmente mais motivadas pela oportunidade de trabalho (o que é escasso), foram fundamentais para o oferecimento de melhores informações sobre a biodiversidade local a um custo menor do que ocorreria numa abordagem científica tradicional. Como exemplos, podem ser citadas as experiências nas florestas de Kinabalu e Sabah, na Malásia. Pesquisadas há mais de um século por botânicos, estas florestas foram incluídas em um projeto que visava aumentar as informações sobre espécies de palmeira entre 10 e 19 anos. Os resultados obtidos foram significativos e mais rápidos por causa desta participação direta dos comunitários locais. O Programa de Inventário Nacional da Biodiversidade da Costa Rica (INBio), por seu turno, introduziu a participação comunitária para a catalogação de aproximadamente 3 milhões de espécies de insetos, o que foi concluído em 13 anos. Na Área de Conservação Guanacaste, foram registrados 200 mil lagartos de 3 mil espécies em duas décadas com a colaboração indispensável dos comunitários. Esta participação local também foi essencial para a especificação de 1010 espécies de insetos mastigadores de folhas de 59 espécies de plantas lenhosas na Nova Guiné. Tal trabalho consumiu apenas 3 anos, surpreendentemente.

Estas colaborações locais favorecem a qualidade dos dados, uma vez que os comunitários podem acompanhar por mais tempo a evolução e deslocamento das espécies biológicas. Ademais, a integração entre cientistas e comunitários se consolida à medida que trocam suas informações, experiências e concepções sobre a conservação da biodiversidade. Os biólogos podem modificar bastante suas opi- 
niões, a princípio mais teóricas, tornando-se melhores observadores e conhecendo com mais detalhes as realidades. Por sua vez, os comunitários têm a oportunidade de conhecer mais profundamente a complexa riqueza em que se inserem, ampliando sua consciência ecológica. Estas pesquisas, por todas as razões expostas, são consideradas como mais eficazes para fins de monitoramento. Entretanto, limitações materiais e culturais são obstáculos ao pleno funcionamento desta parceria. Primeiramente, as imensas precariedades em que vive grande parte das comunidades locais dificultam muitas vezes o desenrolar deste trabalho, o que requer maior presença do Estado com a prestação de serviços sociais e econômicos. Some a isso a adaptação de pesquisadores à realidade local como outro desafio para a qualidade da pesquisa.

Presume-se que a inclusão das comunidades locais na gestão das áreas protegidas e atividades de monitoramento é tendência a se propagar, especialmente nos países tropicais ou em desenvolvimento (SHEIL e LAWRENCE, 2004). Entretanto, o envolvimento comunitário se relaciona fundamentalmente ao retorno em benefícios sociais e geração de renda (DANIELSEN et al, 2007), pois não se pode ignorar que a qualidade de vida é uma das condições importantes para o empenho pela conservação ambiental. Há autores que vêem nesta modalidade de monitoramento a sua importância no fato de que ela pode ser alternativa aos programas governamentais tradicionais dos países em desenvolvimento que apresentam elevada corrupção. Neste contexto, a participação comunitária reduziria incidência de malversação de recursos, apesar da lacuna de estudos comprobatórios a respeito (AFRIDI, 2008).

Observa, no entanto, Jacoby et al (1997) por outro lado, que o monitoramento comunitário também possui limitações. Um elemento estaria associado à própria qualidade dos dados coletados, sobretudo nos momentos de registro e tratamento dos dados coletados no sistema formal de monitoramento. Esta dificuldade ressaltada exige permanente capacitação dos comunitários. Outrossim, a inconsistência de recursos financeiros e de cumprimento de prazos da parte dos gestores promove desinteresse entre comunitários (WHITELAW et al, 2003).

O processo do monitoramento comunitário ganhou força com os programas participativos de gestão dos recursos naturais. A variação das espécies a serem protegidas, bem como sua destinação, também molda esta cooperação entre cientistas e comunitários. A maioria dos sistemas comunitários de monitoramento é financiada por agências oficiais internacionais. Estes sistemas avançam para serem sistemas cada vez mais especializados que incorporam aspectos dos conhecimentos e das formas de uso dos recursos naturais e da biodiversidade das populações locais pelos sistemas tecnológicos gerados pelo conhecimento científico. Este processo é estimulado pela descentralização da gestão dos recursos naturais a fim de incluir, sobretudo, as populações locais. Esta proposta pretende ser hegemônica, pois vislumbra maior abrangência e profundidade para o desenvolvimento do monitoramento. (GARCIA e LESCUYER, 2008).

Quatro razões são apresentadas para o rápido crescimento do monitoramento comunitário em termos internacionais: a) reduzida capacidade dos governos para monitorar os ecossistemas, em função dos cortes orçamentários expressivos para programas e atividades ambientais; b) atividades de monitoramento realizadas pelos governos findam inadequadas para abordar questões ambientais complexas e emergentes; c) reconhecida da necessidade de envolver as partes interessadas e os cidadãos em processo de planejamento e gestão; d) desejo dos comunitários em adentrar neste processo. A parte educacional desta inovação está justamente nesta responsabilidade que os comunitários assumem para a gestão da conservação dos recursos naturais (WHITELAW et al, 2003, p. 412).

Desde a Conferência de Johanesburgo de 2002, a comunidade científica vem se mobilizando, em escala global, a favor do envolvimento das populações locais, sobretudo rurais, na gestão dos recursos naturais. Nos países tropicais, mecanismos que viabilizem a transferência de importantes decisões para este objetivo vão sendo disseminados. A inserção desta nova lógica corresponde, igualmente, a uma importante condição das entidades doadoras de recursos (GARCIA e LESCUYER, 2008).

Danielsen et al (2003), ao abordar criticamente os trabalhos do monitoramento convencional, menciona duas limitações encontradas: a ausência de objetivos articulados e a negligência sobre a estimação da diversidade biológica. O trabalho de monitoramento, normalmente, exige muitos investimentos. Estes investimentos envolvem a presença de pesquisadores, utilização de meios eletrônicos muitas vezes caros e devem ser adaptados a cada realidade. Neste sentido, Danielsen et al (2007) informa que os países desenvolvidos utilizam largamente o monitoramento realizado por cientistas profissionais para avaliar as mudanças populacionais e orientar a gestão das áreas definidas como de conservação prioritária. Ademais, os autores ressaltam que os resultados do monitoramento devem 
gerar uma análise sobre os objetivos traçados pelas políticas globais dirigidas para a biodiversidade. Contudo, é pontuado pelo autor que, nos países em desenvolvimento, nos quais se verificam as maiores concentrações de biodiversidade, os custos dos sistemas tradicionais de monitoramento são elevados, inviabilizando seu financiamento pelos orçamentos públicos locais. Consoante esta tese, nestes países se faz mister que a gestão das áreas protegidas recorra a abordagens alternativas de monitoramento como o compartilhamento da coleta de dados com as populações locais, majoritariamente rurais, que vivem nestas regiões protegidas. Esta aproximação potencializaria os resultados. Assim, a inovação proveniente do monitoramento comunitário pode representar menor impacto financeiro, além de ampliar o alcance do monitoramento devido ao conhecimento local das espécies possuídas pelas populações locais.

Esta inovação metodológica também é fruto do aprofundamento do debate global acerca da necessidade de sistemas participativos e de avaliação de iniciativas voltadas para o desenvolvimento. As experiências de monitoramento comunitário se disseminaram nas últimas duas décadas. Estão em processo de construção, requerendo o envolvimento de todos os comunitários para que realmente dêem resultados. Aspectos como negociação, resolução de conflitos e empoderamento comunitário são vitais para o desempenho do monitoramento comunitário (GAVENTA, CREED e MORRISSEY, 1998). A operacionalização dos sistemas de monitoramento comunitário possui 5 (cinco) princípios:

Tratam os bens e serviços que a comunidade origina do ecossistema que está sendo monitorado; Os benefícios para as populações locais excedem os custos;

Conflitos políticos entre gestores governamentais e as comunidades não limitam o envolvimento dos atores locais;

Os dados são arquivados, analisados e acessíveis localmente;

O monitoramento baseia-se em instituições tradicionais existentes e outras estruturas de gestão, tanto quanto possível. No entanto, o sistema pode não funcionar corretamente quando a necessidade de melhor governança em contraposição a práticas tradicionais e antidemocráticas. (DANIELSEN et al, 2005 apud GARCIA e LESCUYER, 2008).

Pelos princípios expostos, pode-se certificar que a participação dos comunitários não deve ser apenas na execução do trabalho de pesquisa, mas na capacidade de retornos que poderão ser convertidos em benefícios para os próprios comunitários. Problemas políticos, em certas ocasiões, são verdadeiros óbices ao funcionamento adequado de programas inspirados nesta metodologia. Em contextos onde mudanças são difíceis de acontecer, marcadas por péssimas relações entre comunitários e autoridades públicas e, sobretudo, onde as necessidades coletivas em termos de saúde, educação, geração renda, entre outras, são significativas, a propensão de programas e sistemas participativos para a gestão de áreas protegidas fracassarem é maior (DANIELSEN et al, 2007).

\subsection{O ProBUC como proposta de monitoramento comunitário para gestão das unidades de conservação.}

Cada vez mais, instituições patrocinadoras de gestão de biodiversidade passam a exigir, no atual contexto, o monitoramento comunitário na gestão de áreas protegidas, a exemplo da Organização das Nações Unidas para a Alimentação e a Agricultura (FAO), Banco Mundial e a Agência Norte-Americana para o Desenvolvimento (USAID) (DANIELSEN et al, 2000). No caso do ProBUC, seu financiamento é custeado pelo governo do estado do Amazonas juntamente com outras instituições, propondo-se ser um monitoramento com forte presença comunitária. Esta forma alternativa de monitoramento consiste em envolver nesta execução diretamente gestores e comunitários que vivem em meio à biodiversidade. (ABBOT e GUIJT, 1998, p.97).

O monitoramento executado pelo ProBUC apresenta 06 componentes, que correspondem aos recursos e espécies a serem monitorados: fauna, trânsito de embarcações, quelônios aquáticos, recenseamento, pesca comercial e jacarés. Paralelo a esta finalidade, o ProBUC pretende estimular a participação comunitária na gestão da unidade de conservação. Parte-se do pressuposto de que tal participação é indispensável para uma efetiva gestão ambiental, a qual consiste, como já mencionado, viabilizar por meio de acordos entre os grupos sociais envolvidos no processo de gestão de unidades de conservação as condições para a conservação ambiental que deve estar associada ao desenvolvimento socioambiental. 
O monitoramento, na modalidade comunitária, estaria em consonância com a gestão das unidades de conservação conforme o diploma legal mencionado. A implantação de unidades de conservação, cujo funcionamento adequado depende da participação das populações que vivem em seu interior, é uma exigência legal, vide que a própria legislação específica - o Sistema Nacional de Unidades de Conservação - estabelece no artigo 4, concernente às "diretrizes", inciso III, que "[...] assegurem a participação efetiva das populações locais na criação, implantação e gestão das unidades de conservação[...]".

O objetivo da participação comunitária no ProBUC pretende ampliar a conscientização destas populações sobre o valor e o significado da proteção da biodiversidade, especialmente das espécies que estejam em risco de sobrevivência. Questiona-se se este monitoramento realmente consegue corresponder aos seus objetivos, se produzem dados que realmente podem ser utilizados como informações no auxílio da gestão da unidade de conservação. Entre 2005 e 2010, este programa está sendo implementado em duas unidades de uso sustentável, que são as reservas de desenvolvimento sustentável do Uatumã, e do Uacari e em uma unidade de proteção integral, o Parque Estadual do Rio Negro Setor Norte.

$\mathrm{Na}$ reserva do Uacari, que se localiza no rio Juruá, município de Carauari, o ProBUC está implantado desde 2005, com a participação do Instituto Chico Mendes (ICMBio) e da Universidade Federal do Amazonas, tendo o aporte financeiro da Fundação Moore, por meio do Programa "Áreas Protegidas da Amazônia (ARPA)" e do Projeto Corredores Ecológicos. De acordo com Batista et al (2008, p.117) possui uma área de 633.000 hectares, sendo habitada aproximadamente por 236 famílias localizadas em 32 comunidades. Na reserva do Uatumã e no parque Rio Negro Setor Norte este Programa está em implantação desde 2008. Na reserva Uatumã apresentava, por meio de seu plano de gestão elaborado em 2007, cerca de 257 famílias. Nesta unidade de conservação, que envolve os municípios de Itapiranga e São Sebastião do Uatumã e tem 424.430 hectares, o ProBUC é implementado em parceria com o Instituto de Conservação e Desenvolvimento Sustentável do Amazonas (IDESAM) e com o Centro de Pesquisas e Proteção de Quelônios Aquáticos (CPPQA). No parque estadual Rio Negro Setor Norte, localizado na margem direita do rio Negro, no município de Noivo Airão, com uma área de 146028 hectares, vivem aproximadamente 230 famílias. Nesta localidade, o programa é desenvolvido em parceria com a Fundação Vitória Amazônica, como informa Borges et al (2008, p. 16).

Há de se destacar que esta inserção do monitoramento comunitário permite localizar as oportunidades e potencialidades a serem ajustadas ao plano de manejo da unidade de conservação, permitindo, assim, aproveitamento econômico e familiar dos comunitários por meio do manejo de espécies aptas. Esta atividade participativa requer mobilização e sensibilização de todos comunitários, contribuindo para o fortalecimento de seus vínculos e, por conseguinte, viabilizando sua influência na condução da gestão da unidade de conservação. De acordo com Junior et al (2011, p.13), este trabalho do monitoramento comunitário possibilita:

\footnotetext{
Empoderamento das comunidades para atuação mais participativa na gestão da UC;

Valorização do conhecimento e dos modos de vida tradicionais;

Integração entre o conhecimento científico e o conhecimento tradicional;

Geração de benefícios diretos aos comunitários monitores;

Capacitação técnica dos comunitários;

Sensibilização sobre questões ambientais;

Redução de custos para a realização das atividades de monitoramento.
}

Coordenado por uma equipe técnica, o PrOBUC, inicialmente, prevê uma seleção de monitores comunitários, realiza um curso de dois módulos (nivelamento e especialização), onde os conhecimentos tradicionais são associados aos formulários e à metodologia das atividades de campo (JUNIOR, et al, 2011, p. 34). O monitoramente efetuado pelos comunitários é também monitorado. O CEUC possui uma metodologia chamada "ciclo PDCA" P - P (plan) planejar; D (do) executar; C (check) checar; A (act) agir corretivamente. Esta metodologia consiste em checar os dados provenientes das atividades comunitárias, ajustá-las e reencaminhá-las para serem socializadas e discutidas em fóruns com os comunitários, gestores e pesquisadores do Programa. Neste sentido, as oficinas de avaliação participativa, que ocorrem teoricamente a cada 06 meses, e as oficinas de trabalho, cuja frequência é maior, são os espaços onde se propõe a interação entre todos os atores envolvido na construção do ProBUC se dá. Conhecimentos tradicionais e científicos promoveriam, em tese, um diálogo com vistas 
a uma permanente avaliação do trabalho desenvolvido com base nos resultados alcançados.

3.3 Reuniões de avaliação do ProBUC

A realização da primeira reunião (workshop) sobre avaliação do ProBUC gerou um relatório intitulado "ProBUC: avaliação e aplicabilidade à gestão de UCs", realizado entre os dias 23 e 24 de novembro de 2010, em Manaus. Consistiu numa ocasião para especialistas, gestores e comunitários exercerem uma socialização e nivelamento sobre suas compreensões e expectativas quanto a este programa. No período de 22 e 23 de outubro de 2013, ocorreu a segunda avaliação do ProBUC, realizado pelo Centro Estadual de Unidades de Conservação(CEUC), órgão estadual do governo amazonense a que a gerência deste programa de monitoramento é vinculada. Este segundo workshop se realizou numa sala de aula do Instituto Nacional de Pesquisas da Amazônia (INPA). Tratou-se de uma reunião técnica, sem a presença de comunitários, sob a justificativa que havia a necessidade de um público menor e qualificado para um debate mais aprofundado acerca dos dilemas por que passa o programa. O objetivo foi o de identificar as fraquezas e sugerir recomendações. Compareceram ex-técnicos que trabalharam na sua implantação e gerenciamento, além de outros especialistas que acompanham seu funcionamento. Neste evento de avaliação do ProBUC as fraquezas (limitações) foram elencadas como escassez de servidores do CEUC; tempo determinado do financiamento do Programa pela Fundação Moore; necessidade de gerenciamento de dados; escassez de inovação tecnológica; linguagem inadequada usada pela equipe técnica no relacionamento com os comunitários, incluindo o material didático até então empregado, considerando a baixa escolaridade da maioria dos moradores das UCs envolvidas; dificuldade dos comunitários identificarem no ProBUC a finalidade de auxiliar a gestão das UCs, uma vez que a expectativa dominante se relaciona à possibilidade de geração de renda; baixa capacidade analítica dos dados. Algumas das principais recomendações feitas pelos técnicos presentes foram: inserir os conteúdos informativos e formativos do Programa nas escolas existentes dentro das UCs envolvidas; realizar cursos pelo menos uma vez ao ano ou dentro das oficinas de capacitação para tratar de técnicas de comunicação destinadas aos monitores, uma vez que a linguagem atualmente empregada vem comprometendo a compreensão dos objetivos e da missão do ProBUC; divulgação dos resultados pela assessoria de comunicação da Secretaria de Meio Ambiente e Desenvolvimento Sustentável; análises e sumários espaciais dos dados, objetivando a consulta rápida das tendências dos dados e a utilização por parte da própria equipe técnica; política de uso das informações pelos usuários externos, enfocando o potencial de metadados junto às entidades de pesquisa que possam realizar a análise e buscar especialistas que auxiliem nas análises específicas que o Programa demandar; construção de uma gerência de dados que permita uma revisão técnica dos componentes para adequar a atual estrutura de dados.

\section{CONCLUSÃO}

A obrigatoriedade do monitoramento nas últimas décadas para diagnosticar e propor mudanças à gestão de áreas protegidas foi avanço inegável dentro da estratégia global que visa preservar a diversidade biológica ainda existente. Para além de ser uma alternativa barata nos países tropicais, onde seus recursos orçamentários normalmente não comportam este investimento, a construção do monitoramento comunitário como alternativa participativa para preservar a biodiversidade e subsidiar a avaliação da gestão de unidades de conservação é um desafio que exige condições que mobilizem recursos financeiros, técnicos e articulação junto às comunidades locais. Trata-se de uma abordagem que inicialmente estava direcionada à gestão propriamente, até que se constatou que sua eficácia deveria chegar ao monitoramento por causa de seus conhecimentos específicos. No contexto amazônico, dada sua complexidade, mais ainda se torna importante esta parceria. O ProBUC se apresenta como um programa pioneiro no Estado do Amazonas com este objetivo. Sua consolidação passa por permanentes avaliações de seus resultados. No presente, elas demonstram que a busca por seu aperfeiçoamento exige mudanças significativas em sua gestão para que alcance a sustentabilidade financeira, técnica e política. O governo amazonense precisa fortalece os investimentos neste programa para que de fato possa corresponder às finalidades e expectativas geradas. Desta maneira, o ProBUC poderá mostrar novos desafios e perspectivas à construção desta metodologia participativa que visa a preservação da biodiversidade aliada ao uso sustentável dos recursos naturais. 


\section{REFERÊNCIAS}

ABBOT, JoanneGUIJT, Irene. Changing Views on Change: Participatory Approaches to Monitoring the Environment. In: International Institute for Environment And Development, Londres, UK, 1998. Disponível; em: <http://books.google.com.br/books?hl=pt-BR\&lr=\&id=ZeL0o4AF8aAC\&oi=fnd\&pg=PA4\&ots=XT85gftYu\&sig=1jecuGKrhhHiyO8Dts3RvJGQtkI\#v=onepage\&q\&f=false>. Acesso em 03. Jan. 2014.

AFRIDI, Farzana. Can community monitoring improve the accountability of public officials? In: Economic e political weekly. Vol. 43. N. 42, 2008. Disponível em: <http://www.jstor.org/stable/i40010942> Acesso em 11. Nov. 2013.

BATISTA, Gelson da Silva et al. O uso do recurso pesqueiro na reserve de desenvolvimento sustentável de Uacari, Amazonas, Brasil, subsídios para elaboração do plano de gestão. In: CALANDINO, D.et al. (orgs.). Áreas protegidas da Amazônia. Brasília, DF. Ministério do Meio Ambiente, 2008.

BORGES, Sergio Henrique et al .Construindo a gestão do parque estadual Rio Negro setor norte, Amazonas, Brasil.In: In: CALANDINO, D. et al. (orgs.). Áreas protegidas da Amazônia. Brasília, DF. Ministério do Meio Ambiente, 2008.

BRASIL. Lei 9985 de 18 de julho de 2000 Regulamenta o artigo 225, parágrafo $1^{\circ}$, incisos I,II,III e VII da Constituição Federal, institui o Sistema Nacional de Unidade de Conservação da Natureza e dá outras providências. Ministério do Meio Ambiente, Brasília. Disponível em:<http://www.mma.gov.br/port/sbf/dap/ doc/snuc.pdf>. Acesso em 29 maio 2013.

CUNDILL, George; FABRICIUS, Christo. Monitoring in adaptive co-management: towards a learning based approach. In: Journal of environmental management, n. 90, issue 11, 2009. Disponível em: < http://www. sciencedirect.com/science/article/pii/S0301479709001510> Acesso em 27 set. 2013.

DANIELSEN, F. et al. A Simple System for Monitoring Biodiversity in Protected Areas of a Developing Country. In: Biodiversity and Conservation, 2000, v. 9: 1671-1705. Disponível em: <http://journals.cambridge.org $/$ action $/$ displayFulltext?type $=1 \&$ fid $=186888 \&$ jid $=$ ORX\&volumeId $=37 \&$ issueId $=04 \&$ aid $=186887>$ Acesso em 12. Set. 2013.

et al. Biodiversity monitoring in developing countries: what are we trying to achieve? Oryx, 2003 - Cambridge Univ Press.

et al; Monitoring matters: Examining the potential of locally-based approaches. In: Biodiversity and conservation v. 14 Issue 11, pp 2507-2542, Springer, 2005. Disponível em: < http://link.springer. com/article/10.1007/s10531-005-8375-0\#page-1> Acesso em 14 jan. 2014

et al. Increasing conservation management action by involving local people in natural resource monitoring. In: AMBIO: journal of the human environment, v. 36, n. 7, 2007.

GARCIA, Claude A.; LESCUYER, Guillaume. Monitoring, indicators and community based forest management in the tropics: pretexts or red herrings? In: Biodiversity and Conservation, 2008 - Springer. Disponível em: <http://link.springer.com/article/10.1007/s10531-008-9347-y> Acesso em 13 jun. 2013.

GAVENTA, John; CREED, Victoria; MORRISSEY, Janice. Scaling up: participatory monitoring and evaluation of a Federal Empowerment Program. In: New Directions for Evaluation, n. 80, Winter, 1998. Disponível em: <http://onlinelibrary.wiley.com/doi/10.1002/ev.1119/abstract> Acesso em 12 set. 2013.

GLASSON, Jhon; THERIVEL, Riki; CHADWICK, Andrew. Introduction to environmental impact assesment. In: Introduction to environmental impact assessment. The natural and built environment series, fourth edition. UCL Press, Londres, 1994. Disponível em: <http://books.google.com.br/books?hl=ptBR\&l$\mathrm{r}=\& \mathrm{id}=$ NefZAAAAQBAJ\&oi=fnd $\& \mathrm{pg}=\mathrm{PP} 1 \& \mathrm{dq}=.+$ Introduction + to + environmental + impact + assesmen- 
t\&ots=dnHMQq8F_Y\&sig=5uioBsaEifMJQli6VehxHXo2jF8\#v=onepage\&q=.\%20Introduction $\% 20$ to\%20 environmental\%20impact\%20assesment\&f=false > Acesso em 10 jan. 2014.

HOCKINGS, Marc. Evaluating effectiveness - A framework for assessing the management of protected areas. In: Oxford journals, v.53, n.9, 2000. Disponível em:< http://bioscience.oxfordjournals.org/content/53/9/823.short> Acesso em 27 dez. 2013.

JUNIOR, Sinomar F. Fonseca et al. Programa de monitoramento da biodiversidade e do uso de recursos naturais- ProBUC: a experiência das unidades de conservação estaduais do Amazonas. Manaus, Centro Estadual de Unidades de Conservação, 2011.

LAMMERTS van BUEREN EM, BLOM EM. Hierarchical framework for the formulation of sustainable forest managemente standards. In: Tropenbos Foundation, 1997. Disponível em: < http://www.piec.org/ pathfinder/Pathfinder_portal/Instruments_Engl/A3Hierarchical_framework/print/CI_framework_PF.pdf > . Acesso em 23 nov. 2013.

MARINELLI, Carlos Eduardo et al. Ciência, tecnologia e sociedade: sinergia e inovação para construção de conhecimento coletivo sobre UCs da Amazônia Brasileira. In: De olho nas unidades de conservação: sistemas de indicadores socioambientais para unidades de conservação da Amazônia Brasileira. São Paulo: Instituto Socioambiental, 2011.

REBELO, George; PEZZUTI, Juarez. Percepções sobre o consumo de quelônios na Amazônia: sustentabilidade e alternativas ao manejo atual. Ambient. soc., Campinas, n.67, jun. 2000.Disponívelem <http://www. scielo.br/scielo.php?script=sci_arttext\&pid=S1414753X2000000100005\&lng=pt\&nrm=iso $>$. Acesso em 25 set. 2013.

SHEIL, Douglas; LAWRENCE, Anna. Tropical biologists, local people and conservation: new opportunities for collaboration. In: Trends IN Ecology and Evolution, vol. 19, n. 12, 2004. Disponível: < http://www.sciencedirect.com/science/article/pii/S0169534704002915> Acesso em 27 set. 2013.

WHITELAW, Graham, et al Establishing the Canadian community monitoring network. In: Environmental monitoring and assessment, 88, 2003. Disponível em: <http://link.springer.com/article/10.1023/A:1025545813057\#page-1>Acesso em 30 nov..2013. 